\title{
Clustering in Multi-Channel Cognitive Radio Ad Hoc and Sensor Networks
}

\author{
Mustafa Ozger, Member, IEEE, Fatih Alagoz, Ozgur B. Akan, Fellow, IEEE
}

\begin{abstract}
Cognitive Radio (CR) enables dynamic spectrum access to utilize licensed spectrum when it is idle. CR technology is applied to wireless ad hoc and sensor networks, to form Cognitive Radio Ad Hoc Networks (CRAHNs) and Cognitive Radio Sensor Networks (CRSNs), respectively. Clustering is an efficient topology management technique to regulate communication and allocate spectrum resources by $C R$ capabilities of nodes in CRAHNs and CRSNs. In this paper, we thoroughly investigate benefits and functionalities of clustering such as topology, spectrum and energy management in these networks. We also overview motivations for and challenges of clustering in CRAHNs and CRSNs. Existing clustering schemes are reviewed and compared. We conclude by revealing key considerations and possible solutions for spectrum-aware clustering in multi-channel CRAHNs and CRSNs.
\end{abstract}

Index Terms-spectrum-awareness, clustering, multi-channel, cognitive radio, ad hoc networks, sensor networks.

\section{INTRODUCTION}

$\mathbf{E}$ XCESSIVE demand for wireless communications has resulted in spectrum scarcity problem. Although unlicensed spectrum bands are overcrowded, licensed ones are not efficiently utilized. Cognitive radio (CR) stands as a promising solution to overcome this problem by enabling dynamic spectrum access (DSA) for secondary users (SUs) to use idle licensed bands in an opportunistic manner [1], [2]. Hence, CR empowers the communication of SUs by accessing licensed channels without any disturbance to primary users (PUs), which are the licensed users.

SUs coexist with PUs in wireless networks, which are named as CR-enabled wireless networks in general. They can be divided into four domains across two dimensions, as seen in Fig. 1. Firstly, such a network may utilize either a single licensed channel or multiple licensed channels. Secondly, this network may have either a centralized or a distributed network architecture. Centralized CR-enabled wireless networks, i.e., Cognitive Radio Networks (CRNs), have base stations that regulate wireless communications. On the other hand, in distributed ones, there is a flat architecture without any central entity regulating the secondary communication. These

M. Ozger is with Next-generation and Wireless Communications Laboratory (NWCL), Department of Electrical and Electronics Engineering, Koc University, Istanbul, 34450, Turkey (e-mail: mozger@ku.edu.tr).

F. Alagoz is with Department of Computer Engineering, Bogazici University, 34342, Bebek, Istanbul, Turkey (e-mail: alagoz@boun.edu.tr).

Ozgur B. Akan is with Internet of Everything (IoE) Group, Electrical Engineering Division, Department of Engineering, University of Cambridge, CB3 0FA Cambridge, UK (e-mail: oba21@ cam.ac.uk), and also with the NextGeneration and Wireless Communications Laboratory (NWCL), Department of Electrical and Electronics Engineering, Koc University, 34450 Istanbul, Turkey. distributed CR-enabled wireless networks can be termed as Cognitive Radio Ad Hoc and Sensor Networks. Cognitive Radio Ad Hoc Networks (CRAHNs) are flat architectures backed by CR technology, to make the most use of scarce licensed spectrum [1]. Different from CRAHNs, Cognitive Radio Sensor Networks (CRSNs) are employed for sensing applications and consist of nodes with much less computational capabilities and limited battery power [2]. This paper focuses on the domain of multi-channel CRAHNs and CRSNs as seen in Fig. 1.

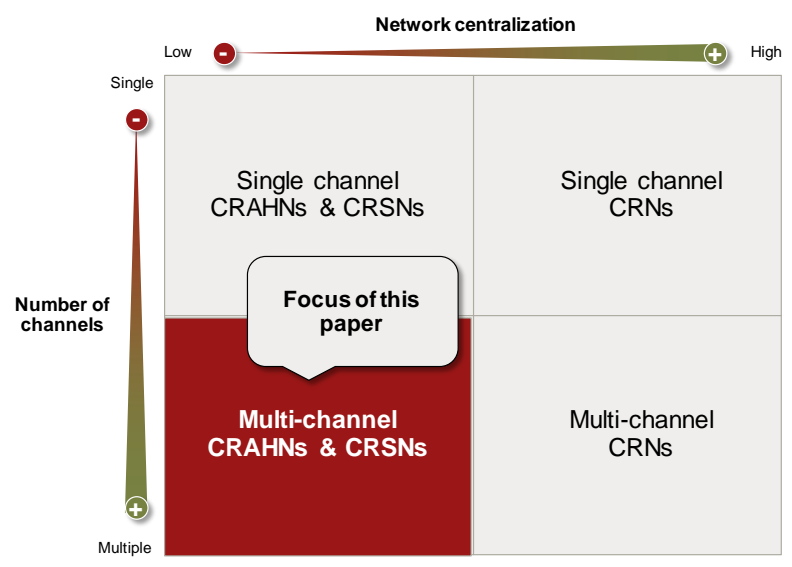

Fig. 1: CR-enabled wireless networks segmented into four domains across two dimensions.

Each SU has DSA functionalities, which are spectrum sensing to determine idle licensed channels, spectrum decision to determine operating channel, and spectrum hand-off to vacate the operating channel if PUs arrive. Hence, SUs use these functionalities to exploit spectrum opportunities, i.e., vacant licensed spectrum bands.

SUs coexist with PUs, and both of them are randomly deployed in the networks. Hence, activities of PUs cause temporal variations in spectrum opportunity for nearby SUs. On the other hand, shadowing, fading and network topology are main drives resulting in spatial variations of idle spectrum bands. Shadowing and fading affect spectrum sensing and cause false alarms and mis-detection of PUs. Network topology differs according to relative transmission range of PUs with respect to that of SUs. There are two network topologies for CRAHNs and CRSNs, which are Topology I and Topology II [3]. In Topology I, there are PUs in the network operating in different licensed channels. However, the transmission range of PUs is larger than that of $\mathrm{CRs}^{1}$ such that PU network

\footnotetext{
${ }^{1} \mathrm{SU}$ and $\mathrm{CR}$ are used interchangeably throughout this paper.
} 


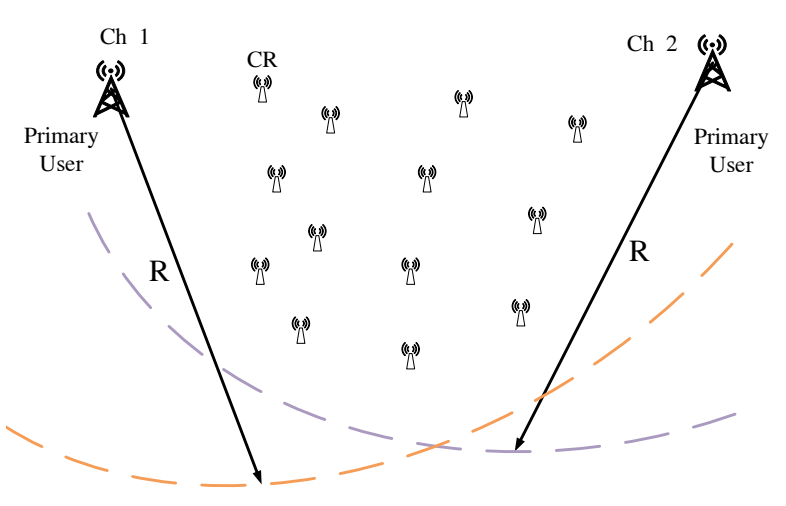

(a) Network topology I

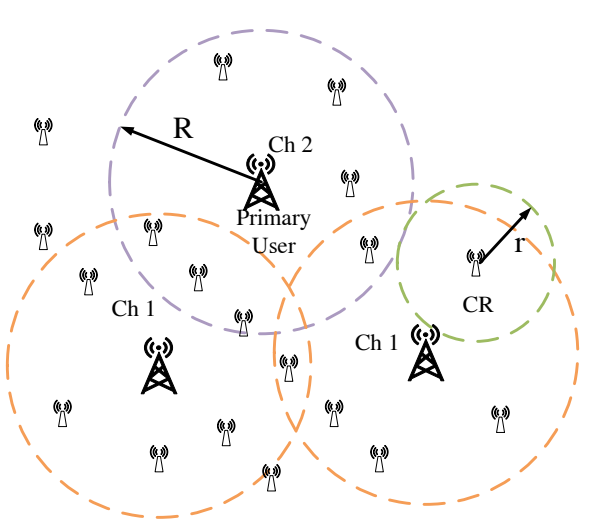

(b) Network topology II

Fig. 2: Network topologies: (a) Topology I, (b) Topology II.

covers secondary network $(R>>r)$ as seen in Fig. 2(a). According to licensed channel usage activities of PUs, there would be temporal variations in spectrum opportunity. Also, there would be spatial variation in vacant licensed bands due to the effects of shadowing and fading. Hence, there would be spatio-temporal variations in the available licensed spectrum bands of CRs. The vacant channel lists of CRs may differ although two PUs using channels 1 and 2 in Fig. 2(a) cover all the CRs.

In Topology II, the transmission range of PUs is comparable with that of CRs $(R>r)$ as seen in Fig. 2(b). Hence, even if the all PUs use their licensed spectrum, there would be spectrum opportunities for the CR nodes located outside of the transmission ranges of PUs. Furthermore, time variations in the channel use of the PUs result in the temporal changes in the spectrum availability of CRs located inside the transmission range of them. This model causes a very dynamic radio environment.

Proposed networking solutions must consider coordination among SUs for the communication in multi-channel CRAHNs and CRSNs. Clustering is a fundamental solution that can be used to manage and regulate communications in distributed wireless networks [4]. It groups neighboring nodes logically in such networks. Each of these group is called a cluster. In a cluster structure, there is a cluster-head that organizes the communication between its cluster members (intra-cluster communication) and among other clusters (intercluster communication). Hence, distributed networks benefit from clustering by imposing cluster-heads to enhance communication performance in the network by achieving stability and supporting cooperation [4]. CRAHNs and CRSNs also benefit from clustering to coordinate spectrum-aware communication in this highly dynamic radio environment due to PU activities for Topology I and Topology II. Hence, clustering needs consideration of common idle licensed channels between cluster members CRAHNs and CRSNs in addition to physical proximity of cluster-head and cluster members. Hence, this type of clustering is called spectrum-aware clustering. Due to this feature, clustering is used as a functional tool for cognitive cycle functions, medium access control, routing, and managing multi-channel communications. Despite its advantages, some key challenges such as heterogeneous licensed channels and dynamic change in vacant channels in the networks must be carefully considered to propose clustering solutions.

This paper is organized as follows. Functionalities of clustering are explained in detail in Section II. Section III motivates the clustering and explains challenges thereof. Existing clustering schemes are presented and compared based on their implementability and offered features in Section IV. We present design considerations and potential clustering solutions for CRAHNs and CRSNs in Section V. Finally, Section VI concludes our paper.

\section{Functionalities of Clustering in CRAHNS AND CRSNS}

This section elaborates how clustering can be employed for various functionalities and their correspondence with network operations. Such functionalities broadly relate to three operation areas, which are sensing, network layers and DSA operations as seen in Fig. 3. Detailed explanation of each operation areas are given in following subsections.

\section{A. Sensing Operation}

Sensor networks utilize clustering to aggregate gathered information at the cluster-heads by exploiting correlation. Hence, clustering can exploit spatial and temporal correlation of sensor nodes' observations on spectrum sensing. Since observations are correlated in time and spatial domains, both topologies can benefit from clustering for sensing operations. Another benefit of clustering is less packet transmissions due to aggregation and hence less energy consumption. Sensing correlation affects routing, medium access and wireless transmission functionalities due to aggregation.

\section{B. Network Layer Operations}

Clustering provides functionality to routing in wireless ad hoc networks. However, CR brings additional challenges due to dynamic variations in available spectrum bands. Common channel between communicating SUs is an additional constraint to forward a packet. Since clusters are formed to have common channels among its members by exploiting spatial 


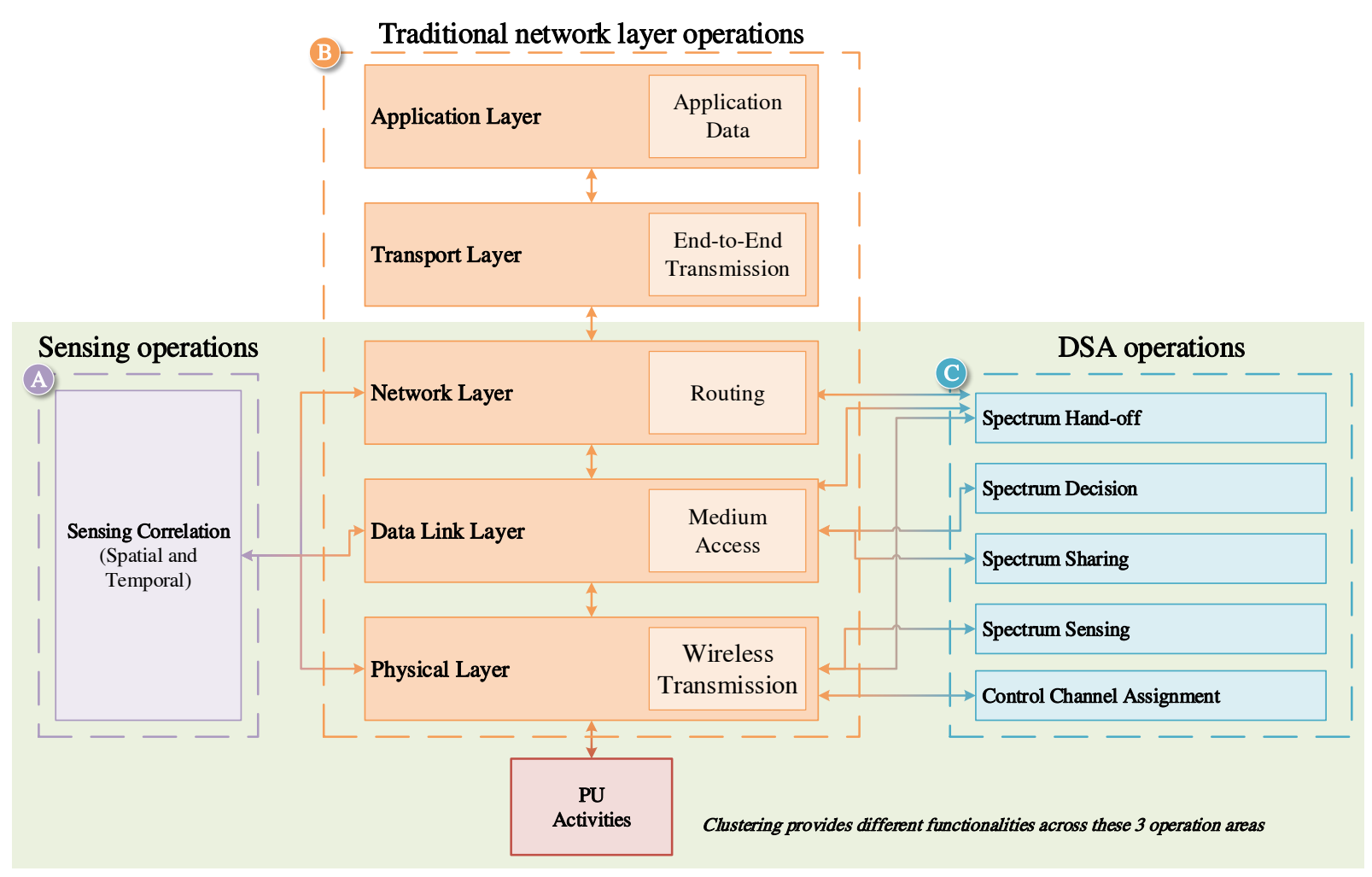

Fig. 3: Functionalities of clustering and their relations with traditional network layers and dynamic spectrum access functions.

and temporal correlation, it provides functionality to routing to reduce overheads while conveying packets from source to destination. Hence, both network topologies can utilize routing functionality of clustering. [5] and [6] are two examples how clustering is utilized for routing in dynamic radio environment. Directed routing from event to sink is proposed by clustering in [5]. Clustering is also utilized to route multimedia packets in CRSNs according to DSA and quality of service requirements [6].

Medium access functionality is also provided with clustering. In dynamic radio environment, one of the most important factor is the PU presence and contention for the idle licensed channel. A medium access scheme can be designed such that every node in a cluster can access the idle channel without contention [7]. The cluster-based medium access provides higher throughput and smaller delays with less energy consumption. Cluster-heads can behave as a coordinator for accessing spectrum after sensing the vacant channels collaboratively. Medium access can be performed with clustering in both topologies to assist spectrum-aware communications depending on spatial and temporal variations of licensed bands.

\section{DSA Operations}

Spectrum sharing and decision operations are CR-specific. Contention towards vacant licensed bands and PU arrivals are two important factors to share spectrum and decide operating band. Since clusters are formed according to common vacant channels, cluster-head decides the operating communication channels for intra-cluster communication that contributes to spectrum decision. For instance, clustering can be utilized to decrease the interference among SUs by power and subchannel allocations [8]. The clustering is beneficial for both of the topologies while sharing and deciding the operating spectrum band.

Spectrum sensing is one of the DSA operations. The nodes in a cluster send their observation about the spectrum to the cluster-head for making local decision about the vacancy in licensed channels. The cluster-head behaves as a fusion center to determine the PU presence, which affects physical layer of the network. The nodes in the cluster cooperate to reach a consensus for more reliable spectrum sensing. Although clustering can be utilized for spectrum sensing in both topologies, it is mainly used in Topology I due to less spatial variations. Due to shadowing, fading and imperfections of spectrum sensing, CRs within clusters cooperate to determine vacancy of the spectrum bands in Topology I.

Availability of common control channel throughout the network is another problem in CRAHNs and CRSNs. Clustering functionality provides local control channels among cluster members. Control messages among cluster members are transmitted through these control channels [9]. Since Topology II causes highly dynamic spatial and temporal variations, control channel assignment operation is more suitable for Topology II.

\section{Motivations And Challenges of Clustering in CRAHNS AND CRSNS}

Clustering is an efficient method to organize the networks and to impose local coordinators so that spectrum-aware communications can be performed in a structured way. Hence, 
we describe key motivations and challenges of clustering in CRAHNs and CRSNs in detail.

\section{A. Motivations of Clustering}

Clustering forms logical groups to better utilize the resources in ad hoc networks. It becomes more beneficial for ad hoc networks with DSA since spectrum-aware communication can be performed efficiently by grouping neighbor nodes according to their idle channels. Accordingly, we itemize the motivations of clustering as follows.

- Coordination between nodes: In each cluster, there is a cluster-head that controls the communication within its group. Furthermore, the neighboring cluster-heads may communicate directly via their common channels or via multiple hops through their members. The clusterheads can be considered as virtual backbones regulating the spectrum-aware communication between SUs, which provides coordination among the SUs.

- Scalability: Ad hoc and sensor networks have high network density so that protocols and the solutions in these networks may not be scalable. Furthermore, the dynamic change in the spectrum availabilities of the nodes deteriorates scalability in proposed solutions due to spectrum-aware communications of large number of nodes in the network. On top of homogeneous network, clustering imposes virtual nodes, i.e., cluster-heads, to improve scalability of the network [4].

- Improvement in network performance: Neighboring nodes are grouped according to their idle licensed channels in multi-channel dynamic radio environment. If one common channel in the cluster becomes occupied by a $\mathrm{PU}$, the communication in the cluster continue through another common channel. Hence, CRAHNs and CRSNs become immune to PU arrivals on their operating channels due to spectrum-aware clustering, which increases the network performance.

- Cooperation between the nodes: Cognitive cycle functions, which are spectrum sensing, spectrum decision and spectrum handoff, can be managed in a highly structured way with clustering. These complex tasks are performed in a cooperative manner between the cluster members. Since there are common channels among the cluster members, cluster-head can assist cooperation easily. Furthermore, the cooperation between the clusterheads results in better communication performance of the nodes.

- Imposing local control channels: The most important problem in spectrum-aware communication is to assume a common control channel in the whole network. However, this assumption is not realizable due to dynamic behavior of the idle licensed channels throughout the networks, i.e., PU activities. Since clusters are formed within a neighborhood, local common control channels exist in the clusters for regulating the communication between the cluster members.

- Multi-channel communication support: Each cluster has multiple common channels among its members.
Hence, cluster-heads can allocate spectrum resources within cluster member by supporting usage of multiple channels simultaneously.

\section{B. Challenges of Clustering}

Despite the various motivations of clustering in CRAHNs and CRSNs, there exist challenges, which are outlined as follows.

- Multi-channel environment: CR capability offers the utilization of channels owned by PUs. In this type of environment, the clustering is performed according to not only physical proximity but also common channels among the nodes forming clusters. Hence, nodes should be aware of idle licensed channels while clustering and tune to the same frequency band for the communication.

- Dynamic change in availability of licensed bands: One of the most important factor that threatens the stability of clusters is dynamic change in vacant spectrum bands of the clusters. If there is no common channel among the cluster members, these nodes should be re-clustered. Hence, the formed clusters must be robust to the changes in the available spectrum bands. Furthermore, frequent re-clustering causes energy consumption due to control messages for the formation of new clusters.

- Heterogeneity in licensed channels: Characteristics of licensed channels such as bandwidth and carrier frequency may differ in a realistic scenario in multi-channel CRSNs and CRAHNs. This difference may result in different bit rate, transmission range, etc.

\section{Spectrum-AWARE Clustering Schemes}

Having common idle licensed channels among cluster members is a joint requirement for spectrum-aware clustering protocols in literature. However, they have different approaches apart from common spectrum availabilities in neighborhood while forming clusters. We overview recent existing approaches for clustering in dynamic radio environment.

- RObust Spectrum Sharing (ROSS) [10]: This clustering scheme aims robustness of clusters, which means ultimate sustainability of them in case of increased PU activity. The authors in [10] propose a distributed clustering algorithm, which enables the cluster formation by interactions among neighbors of $\mathrm{CR}$ nodes. It consists of two cascaded phases, which are cluster formation and membership clarification. Firstly, cluster-heads are selected according to connectivity vector, which consists of individual connectivity degree and neighborhood connectivity degree. In this phase, common channels between cluster members are guaranteed and the cluster size is controlled. The second phase is for the clarification of cluster membership. The process of membership clarification is realized as a congestion game. The debatable nodes, which are overlapping nodes with different clusters, choose the cluster with highest common channels after joining them.

- Affinity Propagation (AP) [11]: This algorithm applies affinity propagation so that $\mathrm{CR}$ nodes are data points 
TABLE I: Overall comparison of existing clustering schemes in CRAHN and CRSN.

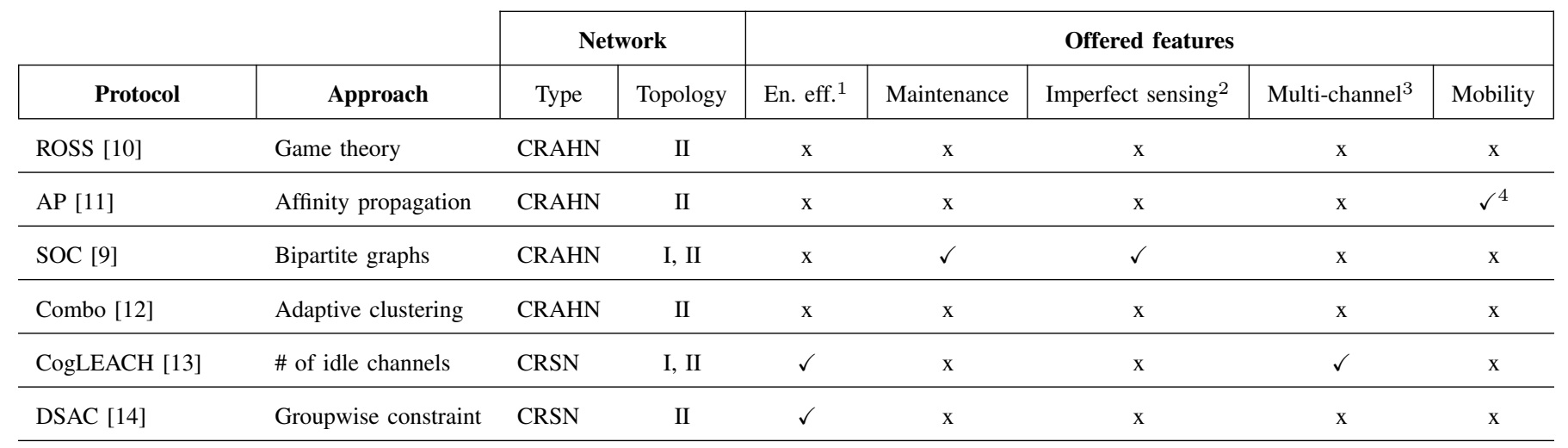

1: Energy efficiency - 2: Resilience to faults in spectrum sensing - 3: Support for heterogeneous multi-channel -4 : Supports only low mobility

and the cluster-heads are exemplars. The main aim is to minimize the number of clusters in the whole network. Similarity measure is the common channels shared by CRs. The objective of the clustering is to have as high number of available channels as possible with other nodes in the cluster. The cluster-heads are chosen with a probability that is determined by the node degree of the CRs.

- Spectrum Opportunity Clustering (SOC) [9]: SOC is a cluster first algorithm. There are three steps for the formation of clusters. In the first step, the nodes know its neighbors and their vacant channels, and they forms bicliques which are maximum edge biclique or maximum one-sided edge biclique. The formed bicliques are broadcast to the neighbors and best biclique is selected according to largest cluster size or edges in the biclique as the second step. In the last step, the nodes with more than one cluster affiliation are removed. The cluster-head is the node with one hop communication distance with the cluster members.

- Combo [12]: In this clustering scheme, nodes know their $k$-hop neighbors and their corresponding vacant channels. All CRs calculate minimum number of common channels with their $k$-hop neighbors. According to this information, a weight is assigned to each CR for cluster-head selection process. The node with highest weight in its neighborhood becomes a cluster-head. These nodes send membership request for their clusters. If a node receives more than one request, it selects the one with the highest weight.

- CogLEACH [13]: In this clustering method, expected number of cluster-heads is fixed and the nodes with higher number of channels more likely to become clusterheads. According to these conditions, every node is assigned a probability to become a cluster-heads. Noncluster-head nodes send join request to the cluster-head with minimum communication cost based on the received signal strength.

- Distributed Spectrum-Aware Clustering (DSAC) [14]: DSAC scheme tries to minimize total energy consumption, which is sum of energy consumed for intra-cluster and inter-cluster communications. Optimal number of clusters are determined according to the average consumed energy. For the distributed algorithm, locally closest pairs are merged for clustering with group-wise available channel constraint.

Table I summarizes the above discussions and gives overall comparison of existing clustering schemes according to the networks and offered features by clustering.

\section{Distributed Clustering In CRAHNs AND CRSNS}

Despite assumption of a clustering scheme existence for spectrum management, medium access control and routing solutions in literature, there are not enough clustering studies to satisfy inherent requirements of CRAHNs and CRSNs. To that end, we determine network requirements and clustering design considerations. Afterwards, we briefly overview potential solutions for distributed clustering approach in CRAHNs and event-driven approach in CRSNs.

\section{A. Network Requirements}

CRAHNs have unique requirements, one of which is managing high fluctuation in spectrum availability due to mobility of CRs and/or PUs. Furthermore, different quality of service (QoS) demands must be satisfied. For instance, multimedia applications require limited delay and lower jitter. Another requirement is limited interference to PUs. If the operating channel change is due to PU activities, there may be interference to the PU, which is undesirable.

There are additional requirements for clustering in CRSNs. First of all, sensor nodes have limited battery power. Hence, the clustering solutions must be energy-efficient. Since clustering requires control signaling for cluster formation and maintenance, proposed clustering solutions should avoid excessive overheads which decrease energy levels of the CRs. In terms of cognitive operations, the proposed clustering should avoid frequent change in operating channel since cognitive cycle functions such as spectrum hand-off and spectrum sensing consume great amount of power. Furthermore, the sensor nodes have limited computational energy. Hence, the proposed clustering approaches should be simple. Energy-efficiency and 
low computational overhead are two additional requirements for clustering solutions in CRSNs.

\section{B. Clustering Design Considerations}

CR operations and distributed network architecture are the main considerations while designing clustering solutions. Design considerations of clustering in CRSNs and CRAHNs differ due to their unique requirements. Hence, we explain common and different design considerations according to the network types as follows.

Common design considerations: The first common consideration is that there needs to be at least one mutual vacant channel among the formed clusters. The cluster nodes must communicate with each other via common channels.

Imperfections in the spectrum sensing is also important since the clustering is performed according to the vacant spectrum bands. The clusters can be formed according to erroneous vacant channel lists of CRs due to poor channel sensing performance. The common method for spectrum sensing is energy detection due to its simplicity [1]. However, this method has imperfections in the sensing process. Hence, the probabilities of mis-detection and false alarm are important for the stability of clusters.

Mobility of the nodes highly affects the heterogeneity in spectrum availability, which directly alters the cluster formation. Cluster-head may lose connection with its members due to mobility of SUs. Hence, the cluster structure changes and the association and disassociation mechanisms should be designed for the incoming and outgoing nodes in the clusters. Furthermore, the mobility of PUs causes more dynamic spatiotemporal changes in spectrum availability of the SUs.

Maintenance of the clusters is another consideration. Highly changing licensed users' activities increase the cost to preserve the clusters. Due to these dynamic effects, some nodes may leave their current clusters or join another one, which results in packet overhead and energy consumption.

Design considerations specific to CRAHNs: The flows in an ad-hoc networks have different QoS levels. This consideration becomes difficult by activities of PUs. Clustering must take them into account to support different QoS levels.

In order to support the better communication quality, another consideration for clustering is heterogeneous licensed channels, which cause different characteristics such as transmission range and bit rate. The channels with less PU arrivals must be utilized in cluster formation to decrease the probability of spectrum hand-off, which increases energy consumption.

Design considerations specific to CRSNs: CRSNs have event-driven type of communication. The nodes report their observations after detection of an event. If the frequency is low enough, there is no need to maintain clusters after the event since they will not be utilized until the next event. Clustering solutions should consider this unique property of CRSNs.

Energy-efficiency is another consideration for CRSNs since the nodes have limited power. Despite the fact that clustering provides energy savings, PU activities may degrade the energyefficiency provided by clustering due to re-clustering and control overhead.

\section{Distributed Clustering Approach for CRAHNs}

Absence of centralized entity in CRAHNs necessitates cooperation between CRs. Clustering provides hierarchical structure on top of ad hoc networks architecture to improve local cooperation in the network. To this end, we explain possible problems and solutions in a distributed clustering approach for CRAHNs.

- There is a general assumption about the existence of common control channel in the whole network. However, this is quite unrealistic due to PU activities. To make it more realistic, this channel is assumed to be a very narrow band channel, which may get congested easily. Hence, the proposed protocols must not rely on the existence of such channel. Channel hopping or rendezvous approaches could be adopted to communicate via same channel to cooperate for the formation of the cluster in the neighborhood. However, this approach has high overhead due to channel switching. A blind rendezvous algorithm is proposed for distributed cognitive radio networks such that communicating nodes do not need a common control channel and available channel information [15]. The proposed algorithm guarantees rendezvous without any need for time synchronization and consumes short time to rendezvous.

- Existing clustering schemes form robust clusters against dynamic available channel variations. Hence, a clustering scheme that maximizes the number of common channels or number of member nodes in clusters should be devised by heuristic algorithms. A balance between the sizes of common channels and cluster members may be established to form robust clusters as in [9]. Apart from the formation of clusters, their maintenance is another issue to be considered in a realistic scenario. Cluster maintenance solutions should adapt to the variations of common channels in the cluster. A cluster-head may reform the cluster with less nodes to achieve common channels. Associated node should not deteriorate the cluster stability by decreasing the number of common channels.

- In addition to the dynamic radio environment, mobility of network nodes makes the cooperation among them more challenging due to connection losses. The proposed clustering algorithms should be mobility-aware. If a cluster-head lose connection with its members, this results in re-clustering that an energy-efficient clustering approach should avoid. To overcome this problem, the node with higher mobility in its neighborhood should not be a cluster-head. The nodes may have weight to measure the mobility of the nodes to select the clusterheads in local neighborhoods. By doing this, it is less likely to re-cluster the nodes since the cluster-head will not be disconnected from its members. Furthermore, the cluster must manage incoming and outgoing CRs to update cluster memberships for the management of network topology. Radio resource allocation in a cluster is degraded by association and disassociation of nodes due to mobility. 
- Intra-cluster communication management should be also taken into account due to spectrum mobility. The operating frequency band of the cluster may become occupied, hence, the cluster members should organize intra-cluster communication accordingly to a new vacant channel with limited delay. Due to spectrum hand-off, CR nodes consume energy. Hence, the cluster channel with the least PU arrival statistics should be chosen as the operating channel to avoid spectrum hand-off. Weighing channels according to PU arrival statistics helps choosing more idle channel for intra-cluster communication.

- Existing clustering schemes assume homogeneous licensed channels such that their channel characteristics are the same. However, in a realistic multi-channel CRAHN scenario, their bandwidth and center frequencies may be different. Hence, characteristics of these channels vary. For instance, spectrum sensing results for the licensed channels differ in such a case. Furthermore, the bit rate of the licensed channels become different. The characteristics of PUs on different channels may vary significantly, which results in very vigorous channel availability. If the network requires more coverage, possible solution to form cluster is to group neighboring nodes with channels having lower center frequencies.

We outline some of the problems in clustering and their respective solutions and research directions carefully to fulfill the objectives of clustering in a multi-channel dynamic radio environment. However, a holistic clustering solution should be proposed to overcome the problems of CRAHNs.

\section{Event-driven Approach for CRSNs}

CRSNs have event-driven traffic such that the resources are utilized when events occur. Hence, event-driven clustering approach is shown to be energy efficient for CRSNs in [5]. Furthermore, this approach solicits clustering in the area between event and the sink since the nodes locating in this region will take part in forwarding event packets to the sink. We explain possible solutions and research directions in distributed clustering approach for CRSNs as follows.

- The event readings by cluster members are aggregated at corresponding cluster-heads by exploiting spatial and temporal correlation between them. Furthermore, the mobility-aware Event-to-sink Spectrum Aware Clustering Protocol (mESAC) [5] considers the mobility of the CR nodes to maintain the clusters. However, a definitive reclustering procedure is not studied. Maintenance of the clusters during the event should be considered carefully by contemplating energy constraint of CRSN nodes. The cluster-heads may decrease the number of its members to increase the number of common channels to avoid a complete re-clustering. Instead, possible re-clustering solutions should consider partitioning of the nodes locally. Hence, nodes which needs re-clustering may be associated with a neigboring cluster which should located in the direction of those nodes. Association processes should be performed with minimal overhead and should not degrade the number of common idle channels of the associated cluster.

- Fast convergence of clustering structures is one of the most important goals to decrease communication overhead of clustering to transport event readings to the sink. Preference on cluster-head or cluster first for designing the clustering algorithm may affect the convergence. Cluster-head first algorithms converge faster due to the fact that cluster-heads associate their neighbors to form the clusters according to vacant licensed channels by fewer iterations. After determining the cluster-heads, they directly forms clusters by sending the clustering request to the neighboring nodes.

- Heterogeneous multi-channel is not addressed in CRSN domain. The heterogeneity causes different channel throughput, communication ranges and error probabilities. Hence, it affects the clustering performance and energy consumption. Channel switching may occur frequently to use channels becoming idle with better characteristics. Hence, possible clustering solutions must include the channels with less PU arrivals and more idle probability, which decreases the possibility of reclustering.

- Possible clustering solutions should also consider connectivity of clusters. The solutions should arrange clusterheads so that neighboring cluster-heads can communicate via common channels via single hop. If there is not a common channel between cluster-heads of neighboring clusters, they may communicate via multiple hops in dynamic radio environment. If the cluster-heads directly communicate with each other, it may lead to collisions and interference to the other SUs and PUs due to higher range communication, and this needs packet overheads to rendezvous on the same channel between two neighboring cluster-heads. Multi-hop communication between clusterheads over member nodes is more efficient in terms of interference to PUs and energy consumption.

\section{CONCLUSION}

This paper investigates clustering in multi-channel cognitive radio ad hoc and sensor networks. To this end, we describe how clustering can provide functionalities for different operation regions and explain its benefits. Motivations and challenges of clustering CRAHNs and CRSNs are outlined. Existing solutions for clustering are studied and compared. Furthermore, we present possible clustering solutions to overcome problems in CRAHNs and CRSNs.

\section{REFERENCES}

[1] I. F. Akyildiz, W.-Y. Lee, and K. R. Chowdhury, "CRAHNs: Cognitive radio ad hoc networks," Ad Hoc Networks (Elsevier), vol. 7, pp. 810-836, 2009.

[2] O. B. Akan, O. B. Karli, O. Ergul, "Cognitive Radio Sensor Networks," IEEE Networks, vol. 23, no. 4, pp. 34-40, Jul. 2009.

[3] M. Ozger and O. B. Akan, "On the Utilization of Spectrum Opportunity in Cognitive Radio Networks," IEEE Communications Letters, vol. 20, no. 1, pp. 157-160, Jan. 2016.

[4] K.-L. A. Yau, N. Ramli, W. Hashim, and H. Mohamad, "Clustering Algorithms for Cognitive Radio Networks: A Survey,' Journal of Network and Computer Applications (Elsevier), vol. 45, pp. 79-95, 2014. 
[5] M. Ozger, E. A. Fadel, O. B. Akan, "Event-to-Sink Spectrum-Aware Clustering in Mobile Cognitive Radio Sensor Networks," IEEE Transactions on Mobile Computing, vol. 15, no. 9, pp. 2221-2233.

[6] G. A. Shah, F. Alagoz, E. A. Fadel, and O. B. Akan, "A spectrumaware clustering for efficient multimedia routing in cognitive radio sensor networks," IEEE Transactions on Vehicular Technology, vol. 63, no. 7, pp. 3369-3380, Sep. 2014.

[7] Y. Xu, C. Wu, C. He, and L. Jiang, "A Cluster-based Energy Efficient MAC Protocol for Multi-hop Cognitive Radio Sensor Networks," in Proc. GLOBECOM 2012, pp. 537-542.

[8] J. Dai and S. Wang, "Clustering-based Spectrum Sharing for Cognitive Radio Networks," IEEE Journal on Selected Areas in Communications, vol. 35, no. 1, pp. 228-237, Jan. 2017.

[9] S. Liu, L. Lazos, and M. Krunz, "Cluster-based control channel allocation in opportunistic cognitive radio networks," IEEE Transactions on Mobile Computing, vol. 11, no. 10, pp. 1436-1449, Oct. 2012.

[10] D. Li, E. Fang, and J. Gross, "Versatile Robust Clustering of Ad Hoc Cognitive Radio Network," arXiv: 1704.04828v1 [cs.GT], Apr. 2017.

[11] K. E. Baddour, O. Ureten, and T. J. Willink, "A distributed Messagepassing Approach for Clustering Cognitive Radio Networks," Springer Wireless Personal Communications, vol. 57, pp. 119-133, 2011.

[12] A. Asterjadhi, N. Baldo, and M. Zorzi, "A Cluster Formation Protocol for Cognitive Radio Ad Hoc Networks," in Proc. IEEE European Wireless Conference, 2010, pp. 955-961.

[13] R. M. Eletreby, H. M. Elsayed, and M. M. Khairy, "CogLEACH A Spectrum Aware Clustering Protocol for Cognitive Radio Sensor Networks," in International Conference on Cognitive Radio Oriented Wireless Networks, 2014, pp. 179-184.

[14] H. Zhang, Z. Zhang, H. Dai, R. Yin, and X. Chen, "Distributed Spectrum-Aware Clustering in Cognitive Radio Sensor Networks," in Proc. IEEE GLOBECOM, 2011, pp. 1-6.

[15] J. Li, H. Zhao, J. Wei, D. Ma, and L. Zhou, "Sender-Jump ReceiverWait: A Simple Blind Rendezvous Algorithm for Distributed Cognitive Radio Networks," IEEE Transactions on Mobile Computing, vol. 17, no. 1, pp. 183-196, Jan. 2018.
Mustafa Ozger (M'17-S'12) received the BSc degree in electrical and electronics engineering from Middle East Technical University, Ankara, Turkey, in 2011, the MSc degree and the PhD degree in electrical and electronics engineering from Koc University, Istanbul, Turkey, in 2013 and 2017, respectively. Currently, he is a postdoctoral researcher at KTH-The Royal Institute of Technology, Stockholm, Sweden. His research interests include wireless communications and Internet of Things.

Fatih Alagoz is a professor in the Computer Engineering Department of Bogazici University. He obtained his B.Sc. degree in electrical engineering in 1992 from Middle East Technical University, Turkey, and his D.Sc. degree in electrical engineering in 2000 from George Washington University, USA. His research areas include cognitive radios, wireless networks, and network security.

Ozgur B. Akan (M'00-SM'07-F'16) received his Ph.D. degree in electrical and computer engineering from the Broadband and Wireless Networking Laboratory, School of Electrical and Computer Engineering, Georgia Institute of Technology, Atlanta, GA, USA, in 2004. He is currently with the Electrical Engineering Division, Department of Engineering, University of Cambridge, UK. His research interests include wireless, nano, molecular communications, and Internet of everything. 